\title{
AS “PRAXIS” E PRÁTICAS QUE CONSTITUEM O TRABALHO ESTRATÉGICO DOS CONSULTORES: A ANÁLISE DO CONTEXTO PORTUGUÊS À LUZ DO CAMPO DE PESQUISA DA ESTRATÉGIA-COMO-PRÁTICA
}

THE PRAXIS AND PRACTICES THAT FOUND THE STRATEGIC WORK OF THE CONSULTANTS: ANALYSIS OF THE PORTUGUESE CONTEXT IN THE LIGHT OF THE SEARCH FIELD STRATEGY-AS-PRACTICE

Renato Lopes da Costa ISCTE - University Institute of Lisbon, Portugal renato_jorge_costa@iscte.pt

Nélson Santos Antonio ISCTE - University Institute of Lisbon, Portugal nelson.antonio@iscte.pt

José Manuel Brás-dos-Santos Universidad de Sevilla - Espanha

Submissão: 21/02/2016

Aprovação: 24/03/2017 


\title{
RESUMO
}

Numa retrospetiva histórica, embora a literatura existente apelide o setor de consultoria de gestão como um setor extraordinário e um fenómeno único no contexto empresarial, sucede que os estudos realizados neste âmbito não trazem para o estado da arte as ações e interações no campo da pesquisa estratégica, nem para o campo de investigação a complexidade social e a ambiguidade causal da visão básica de análise dos recursos. Enfatizando o estudo no domínios $\mathrm{H}$ e $\mathrm{G}$ da tipologia dos nove domínios da pesquisa da "estratégia-como-prática" e a partir de uma análise empírica sob a forma de entrevistas semiestruturadas e questionários aplicados a consultores de gestão e gestores de PME em Portugal, este artigo visa apresentar um conjunto de ações e orientações estratégicas para os consultores de gestão que os possam conduzir eficientemente na direção de maiores índices de desempenho organizacional.

Palavras-Chave: Consultoria de Gestão, Estratégia, Estratégia-como-Prática

\begin{abstract}
In a historical retrospective, although the literature calls the management consultancy industry as an extraordinary industry and a unique phenomenon in the business context, in fact the studies in this area do not bring to the state of the art actions and interactions in the field of strategic research and the research field of social complexity and causal ambiguity of basic view analysis of resources. Emphasizing the study in the fields $\mathrm{H}$ and $\mathrm{G}$ type in nine areas of research "strategy-as-practice" and from an empirical analysis in the form of semi-structured interviews and questionnaires given to management consultants and managers of SMEs in Portugal, this article presents a set of actions and strategic guidelines for the management consultants that can lead efficiently toward higher levels of organizational performance
\end{abstract}

Keywords: Management Consulting, Strategy, Strategy-as-Practice 


\section{As "Praxis" e "Practices" que Constituem o Trabalho Estratégico dos Consultores no Setor de Consultoria de Gestão}

O objetivo deste artigo consiste em compreender onde e como é feito o trabalho de estratégia (Whittington, 1996), com que competências e ferramentas, tendo por referencial a tipologia da estratégia-como-prática apresentada por Jarzabkowsky e Spee (2009). Diversos académicos, (entre eles, Whittington, 1996, 2002), têm investigado a possibilidade de a chave do sucesso organizacional estar contida nos detalhes de nível micro, os quais permitirão uma maior vantagem competitiva por serem invisíveis a terceiros. Por outro lado, regista-se um crescente interesse em pesquisar o como estas práticas são desenvolvidas e como constituem o processo estratégico, também designado por estrategizar, conforme o fazem Whittington, (2002), Wilson e Jarzabkowski (2004), Jarzabkowski e Spee (2009), e Jarzabkowski et al. (2007).

Durante muitos anos, analisou-se a estratégia com base nas ações e nas dinâmicas internas das empresas relacionadas ao desempenho organizacional (Johnson et al., 2007), tendo apenas por atores da estratégia determinados grupos de topo. Tal facto esteve associado à ideia, existente entre académicos e práticos, de que apenas estes grupos podem agir de forma estratégica, o que, segundo Johnson et al. (2003), é explicável como resultado do domínio da microeconomia. Só mais recentemente houve preocupação em estudar o contributo das emoções, motivações e ações no âmbito da estratégia (Jarzabkowski e Spee, 2009), nas vertentes formulação, desenvolvimento e execução da mesma. A perspetiva da estratégia-como-prática surgiu como uma nova abordagem que centra o estudo nas ações e interações no âmbito da pesquisa estratégica e que introduz no campo de investigação a complexidade social e a ambiguidade causal da visão básica de análise dos recursos (Jarzabkowski, 2005), contribuindo em simultâneo para explicação da prática que constitui o processo estratégico (Johnson et al., 2003).

Segundo Jarzabkowski et al., (2007), o termo estratégia-como-prática pode ser definido como uma situação que compreende ações, interações e negociações de múltiplos atores na construção da estratégia e um conjunto de situações práticas que se traduzem posteriormente numa determinada atividade, o que implica introduzir como parâmetros o estudo dos práticos (pessoas que fazem o trabalho de estratégia), as práticas (ferramentas sociais, simbólicas e materiais com que a estratégia é feita) e as "praxis", consideradas como fluxo de atividade em que a estratégia ficará implícita (Jarzabkowski, 2005; Jarzabkowski et al., 2007; Whittington, 2006). Práticos, práticas e as "praxis" são os elementos de análise na pesquisa da estratégiacomo-prática, constituindo o que se denomina de "fazer estratégia" (Jarzabkowski e Spee, 2009), ou "estrategizar" (Jarzabkowski et al., 2007), em que se efetua a análise de quem a faz, o que faz, como o faz, e o que usa, de modo a estudar as implicações desta tríade na conceptualização da estratégia (Jarzabkowski e Spee, 2009), bem como no desempenho organizacional. Neste contexto e no âmbito deste artigo, importou conhecer como agem os consultores estratégicos, que trabalho estratégico realizam, que tipo de interações sustentam este mesmo trabalho e com que recursos (Jarzabkowski, 2005), tendo por propósito identificar as "praxis" dos consultores estratégicos, bem como, as práticas utilizadas em termos das suas rotinas.

Jarzabkowski (2005), identificou dois caminhos tendencialmente utilizados pelos "practitioners" para fazer estratégia ou estrategizar:1) processual, que carateriza de estrategização processual, cuja envolvência remete para o uso formal de práticas administrativas, tais como, planos, orçamentos e análises que são frequentemente associadas ao 
trabalho de consultoria; 2) a estrategização interativa, que consiste nas ações diretas e interações frente a frente, entre gestores seniores e outros atores.

Com base nos caminhos identificados por Jarzabkowski (2005), na identificação dos diferentes papéis dos consultores e nos caminhos normalmente utilizados pelos "practitioners", verificase que os diferentes papéis dos consultores suscitam diferentes expectativas no que concerne às atividades que constituem o trabalho estratégico e às diferentes possibilidades de utilização de práticas estratégicas, conforme assinalado por Ludgreen e Blom (2009) e ilustrado na figura 1.

Em sucinto, Ludgreen e Blom (2009) apresentam quatro arquétipos (O "Chanceler", A "Sua Eminência", O Mordomo, O Servente) os quais tem distintos níveis de acesso aos atores da organização e em consequência também distintos tipos de atividades e focos de trabalhos. A posição de "Chanceler" é a que manifesta maiores oportunidades de acesso aos atores chave da organização, o que implica que grande parte do seu tempo é gasto nos mais diversos tipos de interações, quer de carácter formal e/ou informal de forma a providenciar a construção de uma estrutura de informação que facilmente lhes permita a formulação do seu rumo estratégico, acentuando-se aqui por conseguinte a importância da legitimidade interpretativa dos consultores, construída pelas suas ações, perícias retóricas utilizadas, aparência e relação interpessoal com o cliente e, pela reputação e imagem da própria organização cliente onde realizam o seu trabalho. O "Chanceler" representa desta forma a ilustração de "estatuto" tendo a possibilidade de influenciar gestores e executivos em decisões estratégicas a desenvolver.

Figura 1: A estrategização da estratégia dos consultores com base no seu papel

Strategização Interactiva

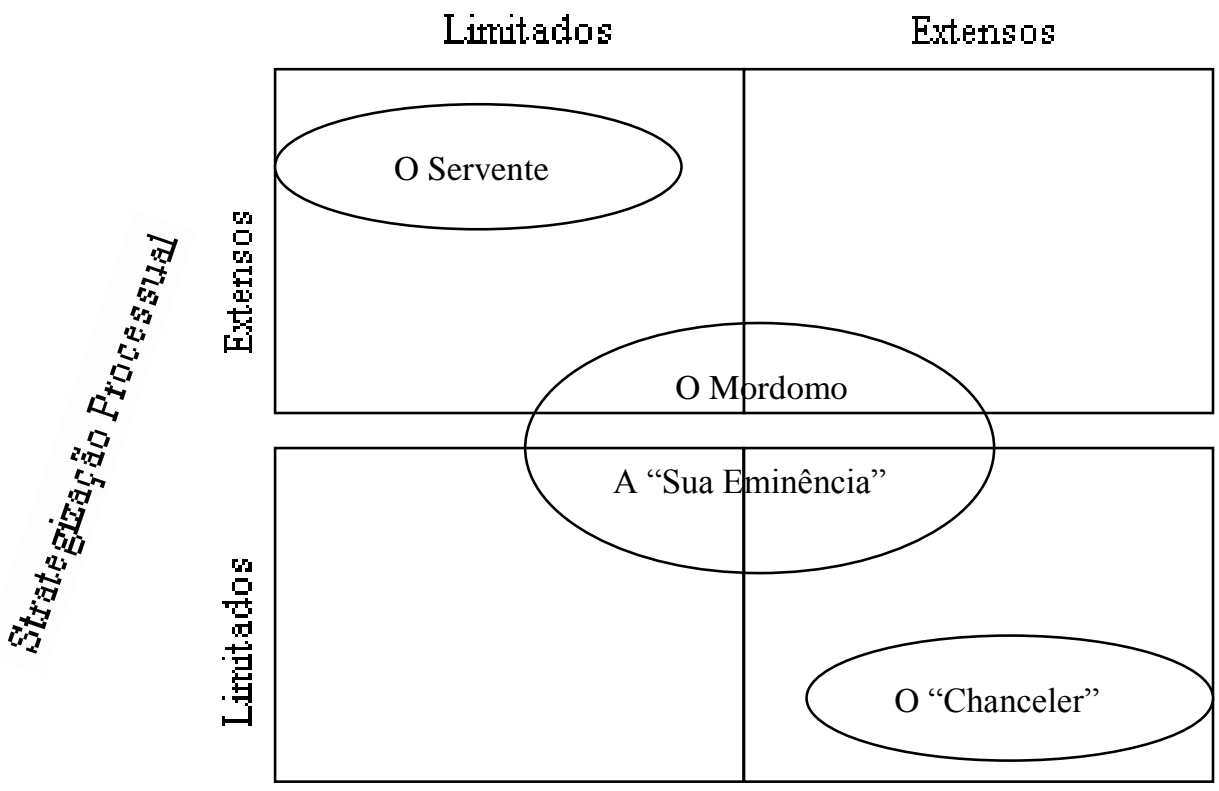

Fonte: Lundgreen e Blom (2009) 
No extremo oposto da figura temos a posição do "Sservente", cuja tendência é manifestamente mais restrita no acesso a atividades que envolvam interatividade. Os consultores que assumem este papel estão nesta conjuntura mais ligados à estratégia do ponto de vista processual, estando normalmente canalizados para o "fazer" sem que possam de forma alguma influenciar estrategicamente quaisquer decisões.

Na linha de Lundgreen e Blom (2009), verifica-se que os diferentes papéis dos consultores podem divergir substancialmente de acordo com as "praxis" e "práticas" que são usadas no desenvolvimento das diferentes atividades estratégicas, o que contribui para a diferenciação dos trabalhos estratégicos nos diferentes grupos que os constituem (Jarzabkowski e Spee, 2009), os quais estão sumarizados na tabela 01 .

Tabela 01: Caraterísticas dos quatro papéis dos consultores e implicações no trabalho estratégico

\begin{tabular}{|c|c|c|c|c|}
\hline & O "chanceler" & $\begin{array}{c}\text { A "sua } \\
\text { eminência" }\end{array}$ & O "mordomo" & O "servente" \\
\hline $\begin{array}{l}\text { Formas de "fazer" } \\
\text { estratégia e uso de } \\
\text { práticas }\end{array}$ & $\begin{array}{l}\text { - Forte confiança na } \\
\text { "estrategização } \\
\text { interativa" } \\
\text { - Perícia retórica e } \\
\text { interações sociais }\end{array}$ & $\begin{array}{l}\text { - Um misto de } \\
\text { "estrategização } \\
\text { interativa } \\
\text { processual" } \\
\text { - Perícia retórica, } \\
\text { interações sociais } \\
\text { combinadas com } \\
\text { trabalho de "back } \\
\text { office" }\end{array}$ & $\begin{array}{lr}\text { - Um misto } & \text { de } \\
\text { "estrategização } & \\
\text { interativa } & \mathrm{e} \\
\text { processual" } & \\
& \\
\text { - Perícia } & \text { de } \\
\text { apresentação, } & \\
\text { recolha de dados e } \\
\text { técnicas } & \text { de } \\
\text { processamento } & \text { de } \\
\text { informação } & \end{array}$ & $\begin{array}{l}\text { - Forte confiança na } \\
\text { "estrategização } \\
\text { processual" } \\
\text { - Acesso a } \\
\text { informações fortes } \\
\text { de dados e técnicas } \\
\text { de processamento } \\
\text { de informação }\end{array}$ \\
\hline $\begin{array}{l}\text { "Praxis": Exemplos } \\
\text { de trabalho } \\
\text { estratégico }\end{array}$ & $\begin{array}{l}\text { - Bom senso para e } \\
\text { conjuntamente com } \\
\text { o cliente } \\
\text { comunicação dos } \\
\text { resultados à } \\
\text { organização cliente }\end{array}$ & $\begin{array}{l}\text { - Bom senso para } \\
\text { e conjuntamente } \\
\text { com o cliente } \\
\text { "por detrás do } \\
\text { palco" } \\
\text { procura e/ou na } \\
\text { própria } \\
\text { iniciativa) }\end{array}$ & $\begin{array}{l}\text { - Assistência } \\
\text { aberta do cliente na } \\
\text { procura com } \\
\text { informações } \\
\text { pontos de vista }\end{array}$ & $\begin{array}{l}\text { - Providenciar de } \\
\text { informação boa e } \\
\text { bem definida e } \\
\text { apresentação desta } \\
\text { como input para } \\
\text { estrategistas de } \\
\text { níveis superiores }\end{array}$ \\
\hline $\begin{array}{l}\text { Resultado(s) } \\
\text { grupo }\end{array}$ & $\begin{array}{l}\text { - Potencial elevado } \\
\text { para influenciar a } \\
\text { orientação } \\
\text { estratégica da } \\
\text { organização cliente } \\
\text { com acesso direto e } \\
\text { aberto a executivos } \\
\text { seniores }\end{array}$ & $\begin{array}{lr}\text { - } & \text { Potencial } \\
\text { elevado } & \text { para } \\
\text { influenciar } & \text { a } \\
\text { orientação } & \\
\text { estratégica } & \text { da } \\
\text { organização } & \\
\text { cliente } & \text { com } \\
\text { acesso direto a } \\
\text { executivos } \\
\text { seniores ainda } \\
\text { que de forma } \\
\text { encoberta }\end{array}$ & $\begin{array}{lr}- & \text { Potencial } \\
\text { moderado para } \\
\text { influenciar a } \\
\text { orientação } \\
\text { estratégica do } \\
\text { cliente, embora } \\
\text { potencialmente } \\
\text { alto em assuntos } \\
\text { com alcance (fins) } \\
\text { definitivos }\end{array}$ & $\begin{array}{l}\text { - Potencial limitado } \\
\text { para influenciar a } \\
\text { orientação } \\
\text { estratégica } \\
\text { cliente }\end{array}$ \\
\hline
\end{tabular}

Fonte: Ludgreen e Blom (2009)

Noutra perspetiva de análise, ainda que a área de pesquisa da estratégia-como-prática possa ser definida como aquilo que as pessoas fazem, analisando a literatura verifica-se que o conceito é mais abrangente do que esta simples especificação sumária baseada em Ludgreen e Blom 
(2009). Tomando, como exemplo, o conceito de Jarzabkowski e Whittington (2008) sobre práticos estratégicos, definidos como os atores diretamente envolvidos na tarefa de fazer estratégia (predominantemente levada a cabo por gestores e consultores) e todos aqueles que indiretamente participam nesse mesmo processo (tais como os media, especialistas em gestão, escolas de negócio e todos os envolvidos no processo de desenvolvimento de políticas que legitimem as "práticas" e "praxis") este enquadramento remete-nos para uma dimensão abrangente, não apenas focalizada num ator de forma individual, mas num conjunto de grupos de "práticos", o que constitui duas dimensões principais da identificação de práticos estratégicos (Jarzabkowski e Spee, 2009). A partir desta unidade de análise os práticos estratégicos podem assim assumir o papel de um ator que age de forma individual (um CEO ${ }^{1}$ como ator individual que interage com outros atores) ou de forma agregada (atribuição de atividades especificas a cada departamento interno na organização resultante de um projeto que se esteja a desenvolver), podendo simultaneamente ser interno (estrutura organizacional interna) ou externo à empresa (consultores, especialistas, câmaras da indústria e do comércio, atores institucionais) dada a localização do prático estratégico face às fronteiras organizacionais (Jarzabkowski e Spee, 2009).

A mesma diversidade dimensional aqui refletida no que concerne à conceptualização dos "práticos estratégicos" é encontrada também ao nível das "praxis". Considerando as definições de "praxis" de Jarzabkowski et al. (2007), Reckwitz (2002) e Sztompka's (1991), este domínio pode ser descrito como a corrente de atividade em que a estratégia é realizada (Jarzabkowski et al., 2007), interligando ações micro de indivíduos e grupos em instituições amplas em que estas ações estão localizadas (Reckwitz, 2002), operacionalizando-se no que as pessoas estão a fazer e no que está a acontecer na sociedade - níveis macro das "praxis" (Sztompka's, 1991). A dimensão de "praxis" é desta forma diluída em ações micro que remetem as "praxis" estratégicas para níveis individuais ou grupais em termos de experiência de um episódio especifico (decisões, reuniões, workshops), em ações meso que explora as "praxis" estratégicas a um nível organizacional e sub-organizacional (mudança de programas, mudança de processo estratégico, mudança de padrões de ações estratégicas - Balogun e Johnson, 2005) e, ações macro, ligadas estas a um nível mais institucional associadas mais frequentemente a ações padronizadas de uma indústria específica (Lounsbury e Crumley, 2007).

Jarzabkowski e Spee (2009) relacionaram os três diferentes tipos de práticos mencionados (indivíduos internos, práticos agregados e práticos agregados externos) e os três diferentes níveis de praxis (micro, meso e macro), propondo a tipologia dos nove domínios da pesquisa da estratégia-como-prática, em que cada um dos domínios representa um possível campo de pesquisa nesta área (Tabela 02).

O domínio A inclui estudos onde os práticos podem ser avaliados em termos individuais a um nível micro das "praxis", o que é considerado por Jarzabkowski e Spee (2009) como um dos mais pertinentes domínios nesta área, de forma a descobrir efetivamente o que os "estrategistas" fazem. Aqui a pesquisa pode incidir sobre a compreensão da associação entre as experiências individuais dos práticos e as "praxis" pessoais estratégicas que lhes estão associadas, procurando obter a sua perceção de contribuição para a formulação de estratégia ou estudando

\footnotetext{
${ }^{1}$ A sigla CEO designa-se como "Chief Executive Officer”, sendo este um cargo na hierarquia operacional das empresas portuguesas normalmente intitulado de diretor executivo ou diretor geral
} 
a relação entre o discurso de um gestor sénior e as "praxis" de uma decisão estratégica específica.

Tabela 02: Tipologia da estratégia-como-prática por tipo de "practitioners" e nível de "praxis"

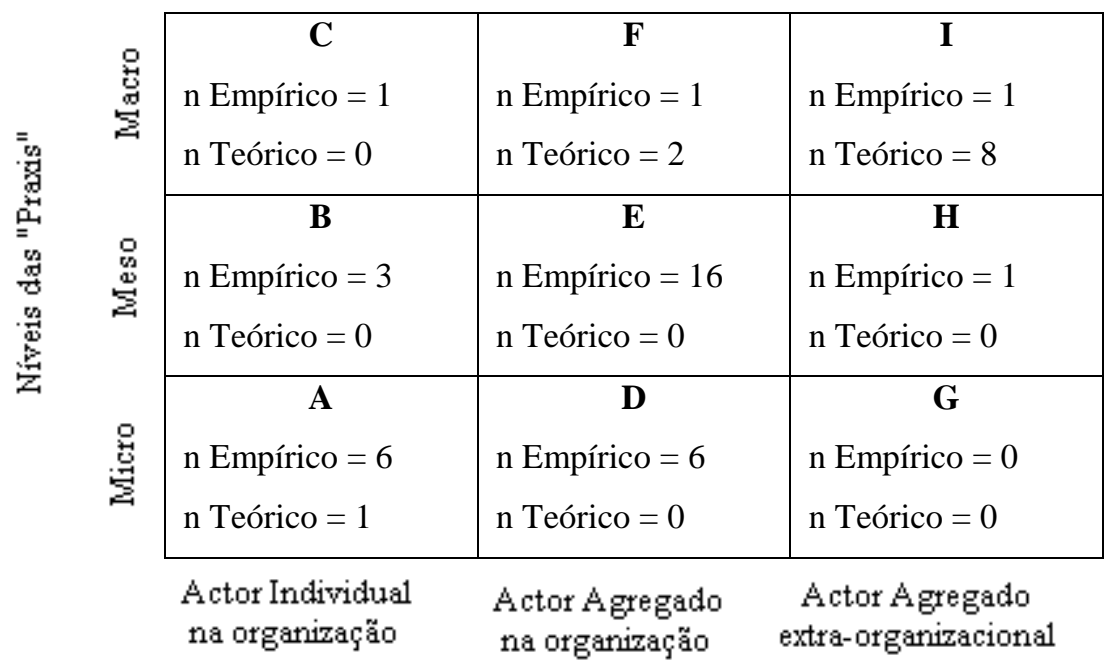

Tipo de Prático
1 - Nesta análise estão apenas implícitos trabalhos teóricos e empíricos identificados com a agenda da estratégia-como-prática

$2-$ Os trabalhos identificados em $n$ Empírico e $\mathrm{n}$ Teórico podem estar incluídos em diferentes domínios e aparecem em mais do que uma análise

3 - Não existem trabalhos identificados em $\mathrm{G}$ e $\mathrm{H}$ pois não têm sido o foco central de nenhum artigo de carácter empírico ou teórico até 2009.

Fonte: Jarzabkowski e Spee (2009)

Domínio B - explora o compromisso individual dos atores em termos das "praxis" organizacionais e sub-organizacionais, tendo em linha de conta por exemplo o que os indivíduos fazem na construção de como se fabrica a estratégia. Este método pode ser utilizado no sentido de estabelecer uma associação entre o que os práticos e as organizações fazem, particularmente em grandes e complexas empresas onde os relacionamentos diretos entre atores e organização são difíceis de substanciar, percebendo por exemplo as implicações e interações de determinados membros de um projeto na implementação de uma nova direção estratégica.

Domínio C - examina a relação entre o indivíduo e as macro "praxis", tendo em linha de conta neste caso o lado institucional da análise, o mercado e as "praxis" da indústria na perspetiva das ações individuais e interações. Como sugerido por Jarzabkowski e Spee (2009) a pesquisa neste domínio poderia incidir sobre como fazem os atores dentro de um escritório regulador em termos da interpretação e resposta relativamente aos "inputs" que recebem dos vários "players" de uma determinada indústria para alterar as regulamentações existentes no mercado, analisando os atores de forma individual em termos de respostas, discursos e capacidade de processamento de informação como forma de perceber a sua interação com o mercado.

Domínio D - examina as relações entre os práticos como atores agregados na organização e as micro "praxis" da estratégia. Assim, com base no conjunto de atores agregados da pesquisa de Sillince e Mueller (2007), uma área de pesquisa neste domínio, tal como sugerido por Jarzabkowski e Spee (2009) poderá estar ligada à tentativa de compreender as interações entre gestores de topo e intermédios dentro de um "workshop" estratégico sobre a forma como interagem na construção das "praxis" aqui envolvidas, ou seja, em termos da conduta e conclusões que poderão daqui resultar, o que nos permitirá trazer para a literatura da estratégia- 
como-prática um nível de análise ligado às interações entre diferentes níveis de atores agregados permitindo simultaneamente perceber as suas implicações.

Domínio E - é idêntico ao anterior, ainda que neste caso os estudos remetam mais para o estudo de padrões de mudança, quer em termos de programas, processos ou ações. Deste domínio resulta a importância que se deve dar às ligações entre as diferentes classes de atores, tais como, gestores intermédios e de topo e ao que fazem as organizações nas diferentes divisões que as compõem. Jarzabkowski e Spee (2009) propõem assim que a chave em termos de investigação neste domínio possa estar ligada ao estudo das "praxis" das diferentes unidades de negócio na implementação de uma mudança organizacional ligada a um determinado programa e à forma como isto pode influenciar as perceções dos diferentes atores agregados no que ao sucesso desta mudança diz respeito.

Domínio F - examina a relação entre atores agregados dentro da organização e as macro "praxis" envolvidas neste contexto ao nível das instituições do setor ou da indústria. Neste domínio realça-se o estudo de Melin e Noordqvist (2007) quando comparam a interação entre gestores de uma empresa de retalho transacional (atores agregados) e atores extra organizacionais na construção e reconstrução de um sistema de governação corporativo. Segundo Jarzabkowski e Spee (2009) uma potencial área de pesquisa no domínio da estratégiacomo-prática poderá estar relacionada com a possibilidade de se poder perceber como os diretores executivos de empresas de retalho podem influenciar as análises nessa mesma indústria (por forma a que se possam construir um conjunto de avultados investimentos nessa mesma indústria), o que permitirá analisar este grupo de atores agregados específicos a um nível macro.

Domínio G - relacionado com o estudo de como os atores extra-organizacionais constroem "praxis" de nível micro. Neste enquadramento, ainda que tenha existido um número considerável de debates sobre como o conhecimento estratégico é construído pelos vários atores (Mazza e Alvarez, 2000), um dos trabalhos que se destacam neste domínio em termos da análise ligada ao campo de agenda da estratégia-como-prática é o de Sturdy et al. (2006), ilustrando o papel dos consultores durante um processo de estratégia examinando as suas interações com os atores organizacionais no decorrer de jantares de negócios. No entanto, ainda que este trabalho tenha implícito uma estrutura teórica e evidências empíricas válidas, este domínio continua a ser limitado em termos de pesquisa, pelo que, "workshops" estratégicos e/ou meetings, decisões estratégicas, micro "praxis", atores externos como os consultores, interpretações e aceitações por parte dos participantes nesses mesmos workshops e/ou meetings, indicam um conjunto considerável de oportunidades para pesquisa futura neste domínio.

Domínio H - foca a relação entre atores agregados extra-organizacionais e as "praxis" estratégicas a um nível organizacional e sub-organizacional. Aqui podem-se salientar no âmbito da consultoria de gestão os trabalhos de Laine e Vaara (2007) e Sminia (2005), ambos focados na influência dos consultores externos em termos do planeamento e implementação de estratégias nas organizações. Uma área de pesquisa neste domínio poderá incidir sobre a influência dos consultores externos ou outros atores agregados extra-organizacionais ao nível das "praxis" estratégicas de uma determinada organização, de forma a examinar o jogo relacional entre o que os atores externos fazem e as ações estratégicas dessa mesma organização.

Domínio I - está relacionado com a associação entre os atores extra-organizacionais e as macro"praxis", ou seja, a ações que estão à partida institucionalizadas em termos de padrão num dado setor ou indústria. O interesse neste domínio tem de facto estado a crescer pelo que o "focus" se deve centrar em termos da compreensão dos diferentes tipos de estratégia em particular, nos recursos estratégicos como práticas institucionalizadas, assim como na forma como essas práticas emergem, são abrangidas e modificadas através da interação entre 
múltiplos atores, o que se pode conseguir através do estudo da institucionalização de tipos particulares de discursos estratégicos dos consultores vs. discursos académicos (Seidl, 2007).

De todos os domínios descritos por Jarzabkowski e Spee (2009) verifica-se que não merececam grande atenção os atores extra-organizacionais, nomeadamente nos domínios $\mathrm{G}$ e $\mathrm{H}$, pelo que esta área de análise terá de ser mais trabalhada em termos de pesquisa a fim de que se possa contribuir com mais estudos empíricos e teóricos que alimentem este campo da estratégia, nomeadamente, da estratégia-como-prática. Em suma, ainda que Lungreen e Blom (2009) tenham providenciado uma interpretação distinta da dinâmica dos relacionamentos existentes entre consultores e clientes, suplantando a imagem tradicional dos consultores estratégicos como poderosos conselheiros em termos das necessidades dos clientes, por uma visão de diferentes variações de dinâmicas e complexos relacionamentos entre consultores e clientes, assim como, providenciado a apresentação dos diferentes papéis que são constituídos e reproduzidos em termos de estatuto, políticas e poder pelos consultores (Werr e Styhre, 2002), um facto que se constata é que as práticas dos consultores de gestão continuam a não ser suficientemente apresentadas em estudos empíricos que comprovem mudanças consideráveis neste aspeto (Jarzabkowski e Spee, 2009), nomeadamente de forma a perceber aquilo que efetivamente estão a fazer, quer ao nível dos artefactos materiais que têm em mão, do seu posicionamento físico nos diferentes episódios estratégicos, das gargalhadas, das frustrações, da raiva, da excitação, da antecipação, do aborrecimento e das próprias manobras e políticas que trazem no pacote de práticas estratégicas (Jarzabkowski e Spee, 2009).

Tal como sugerido por Jarzabkowski e Spee (2009) é vasto o campo de oportunidades para os investigadores académicos nesta matéria, podendo inclusive estes também enveredar no estudo das práticas estratégicas no que às emoções, motivações e às próprias identidades dizem respeito, no estudo das práticas materiais ligadas a estratégias de âmbito tecnológico ou ainda no estudo das "praxis" e dos "practitioners" tais como a tipologia dos nove domínios sugere.

\section{Metodologia}

No que aos meios diz respeito, a presente investigação teve por base um caráter pragmático ou indutivo, e foi conduzida a partir de uma amostra não probabilística por conveniência, constituída de acordo com a disponibilidade e acessibilidade dos elementos abordados (Carmo e Ferreira, 1998), neste caso por consultores de gestão e gestores seniores de PME portuguesas. Neste sentido, foram enviados 300 questionários a PME e 350 a consultores de gestão (onde se incluem 50 consultores independentes) por correio eletrónico. Foram retornados 37 questionários de gestores de PME e 29 de consultores de gestão, totalizando 66 questionários preenchidos com uma taxa de resposta de $10,15 \%$. Todavia, tendo em conta que o interesse desta investigação se centrou em descobrir o significado e representar vivências de múltiplas realidades, foi ainda utilizado como método qualitativo de análise a realização de 17 entrevistas a 9 consultores de gestão seniores e a 8 gestores de PME em Portugal, sendo que a amostra ao nível das entrevistas realizadas, teve um caráter intencional, pois foram selecionados os participantes que melhor representavam o fenómeno investigado em termos de conhecimento.

Em termos da técnica de análise qualitativa utilizada para interpretação dos dados reproduzidos das entrevistas esta traduziu-se numa análise de conteúdo, tentando relacionar as estruturas semânticas (significantes) com as estruturas sociológicas (significados) dos enunciados, de forma a articular a superfície dos textos com os fatores que determinam as suas caraterísticas 
(variáveis psicossociais, contexto cultural e, contexto, processos e reprodução da mensagem) Figura 2.

Figura 02: Categorização e codificação do "corpus" da entrevista para análise qualitativa

$$
\text { Categoría Principal }
$$

\section{Categorías Genéricas}

I.0

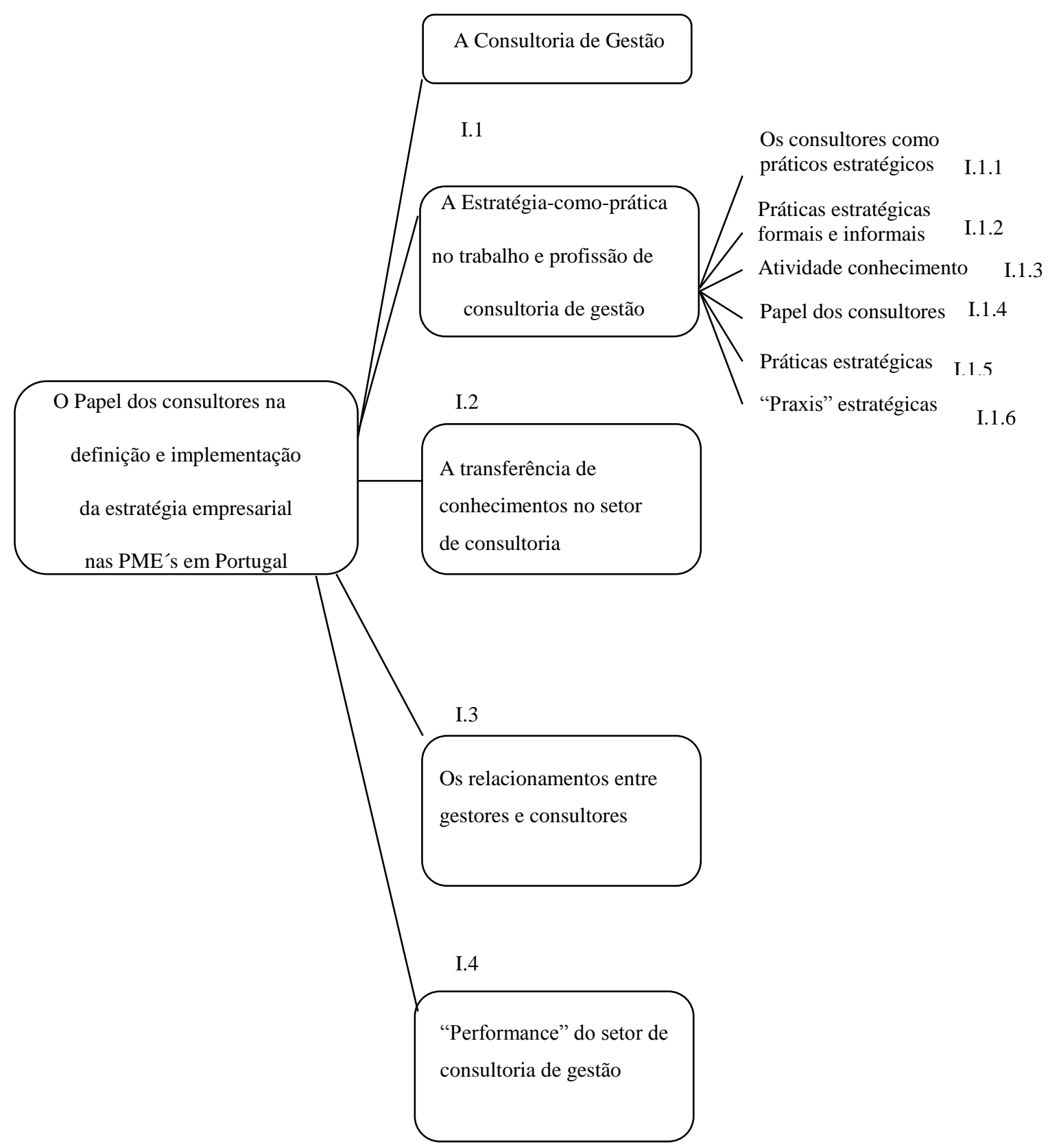

Sub-Categorias 
Em síntese, esta investigação teve por base um conjunto de fontes primárias, a partir da aplicação de inquéritos sobre a forma de 17 entrevistas semiestruturadas a 9 consultores e 8 gestores de PME e, da aplicação de questionários com perguntas abertas e fechadas a 29 consultores e 37 gestores de PME da grande Lisboa, Douro, Leiria, Setúbal, Arquipélago da Madeira, Minho, Beira Alta e Algarve (Portugal), sendo a amostra representativa de um conjunto de inquiridos predominantemente abrangidos por homens (63\%), e cujas as idades têm um valor médio aritmético de 37 anos. Quase todos os inquiridos apresentam uma formação académica de nível superior estando a trabalhar nas suas atuais empresas em termos médios à cerca de 8 anos. De referir ainda que, a amostra ao nível das PME está fundamentalmente ligada à área de retalho e da consultoria aos mais diversos setores de atividade que compõe a área de gestão empresarial.

\section{Resultados da pesquisa}

Contextualizando a análise em termos das "práticas", constatou-se que as práticas identificadas por consultores e clientes como mais importantes para o sucesso do trabalho estratégico em consultoria de gestão foram exatamente as mesmas, ou seja, a capacidade do consultor conseguir influenciar uma decisão estratégica; a sua capacidade em termos de persuasão, influência e assertividade; ter a mente aberta para novas visões ou soluções; a honestidade da sua abordagem; a necessidade da criação por parte do consultor de relações de confiança; a capacidade de se conseguir colocar na pele dos clientes e; o seu grau de flexibilidade e adaptabilidade em termos do processo de decisão (Tabela 3). Nesta análise não se considerou a boa capacidade de interação pessoal tida pelos consultores, por ser considerada uma prática fundamental no desenvolvimento de um qualquer projeto nesta área.

Tabela 3: "Practices" estratégicas consideradas mais importantes por parte de consultores $e$ clientes (gestores) para o sucesso do trabalho estratégico dos consultores na melhoria do seu desempenho organizacional (foram consideradas por cada inquirido as cinco mais importantes - 5 a mais importante até 1 a menos importante)

\begin{tabular}{|l|c|c|}
\hline \multicolumn{1}{|c|}{ Práticas Estratégicas } & $\begin{array}{c}\text { Valores Obtidos } \\
\text { (consultores e } \\
\text { clientes) }\end{array}$ & $\begin{array}{c}\text { Valores } \\
\text { Obtidos } \\
\text { (clientes) }\end{array}$ \\
\hline Capacidade de se colocar na pele dos clientes & 91 & 45 \\
\hline Mente aberta para novas visões ou soluções & 87 & 54 \\
\hline Relações de confiança & 76 & 37 \\
\hline Flexibilidade e adaptabilidade no processo de decisão & 63 & 42 \\
\hline Persuasão, influência, assertividade & 63 & 25 \\
\hline Capacidade de influenciar uma orientação estratégica & 53 & 32 \\
\hline Honestidade de abordagem & 53 & 24 \\
\hline Boa capacidade de interação pessoal & 47 & 18 \\
\hline Conhecimento de um dado setor & 34 & 19 \\
\hline $\begin{array}{l}\text { Congruência entre a exposição de teorias e a perceção } \\
\text { destas }\end{array}$ & 33 & 17 \\
\hline Caráter genuíno de compromisso pessoal & 26 & 13 \\
\hline Inteligência e cultura geral dos consultores & 24 & 5 \\
\hline
\end{tabular}




\begin{tabular}{|l|c|c|}
\hline Perícia na recolha de dados & 22 & 17 \\
\hline Técnicas de processamento de informação & 18 & 9 \\
\hline Contatos influentes & 18 & 0 \\
\hline Entrevista de coleta de dados & 14 & 10 \\
\hline Influência/capacidade persuasão & 14 & 4 \\
\hline Energia e habilidade do consultor & 13 & 9 \\
\hline Utilização da base de dados pelo líder do projeto & 13 & 9 \\
\hline Métodos de abordagem utilizados & 12 & 9 \\
\hline Grau de compromisso pessoal & 11 & 7 \\
\hline Informação e ajuda em conversas de conteúdo & 9 & 7 \\
\hline Estilo e sentido de humor & 9 & 5 \\
\hline Identificação pessoal com o cliente & 8 & 5 \\
\hline Definição de um problema numa reunião & 5 & 4 \\
\hline Perícia de apresentação & 3 & 2 \\
\hline Utilização de uma base de dados & 3 & 3 \\
\hline Estilos de gestão & 2 & 0 \\
\hline Contratação de líder de projeto em conversa banal & 2 & 0 \\
\hline Sentimento de liberdade em termos de pensamento & 2 & 2 \\
\hline Perícia retórica & 1 & 1 \\
\hline
\end{tabular}

Fonte: Elaboração dos Autores

Em termos das "praxis", ainda que os consultores incluam como fator-chave de sucesso o bom senso que devem ter para/e com o clientes, a comunicação de resultados, providenciar e definir informação adequada para consultores seniores. Existem cinco tipos de "praxis" que são tidas conjuntamente como fundamentais para o sucesso de projetos em consultoria de gestão: reunião de processo para início do estudo; o conhecimento dos interesses, valores, atitudes e visões do cliente; a identificação do cliente e do negócio; a reunião semanal ao nível da análise, planeamento e implementação e; a importância das metodologias de trabalho (Tabela 4).

Tabela 4: "Praxis" estratégicas consideradas mais importantes por parte de consultores $e$ clientes (gestores) para o sucesso do trabalho estratégico dos consultores na melhoria do seu desempenho organizacional (foram consideradas por cada inquirido as cinco mais importantes - 5 a mais importante até 1 a menos importante)

\begin{tabular}{|l|c|c|}
\hline \multicolumn{1}{|c|}{$\begin{array}{l}\text { "Praxis" Estratégicas } \\
\text { (consultores e clientes) }\end{array}$} & $\begin{array}{c}\text { Valores } \\
\text { Obtidos } \\
\text { (consultores } \\
\text { e clientes) }\end{array}$ & $\begin{array}{c}\text { Valores } \\
\text { Obtidos } \\
\text { (clientes) }\end{array}$ \\
\hline Metodologias de trabalho & 105 & 47 \\
\hline Conhecer o cliente - interesses, valores, atitudes e visões & 99 & 58 \\
\hline Identificação do cliente e do negócio & 91 & 47 \\
\hline Reunião semanal ao nível da análise, planeamento e implementação & 60 & 25 \\
\hline Reunião de processo para início do estudo & 37 & 25 \\
\hline Bom senso para/e com o cliente & 34 & 16 \\
\hline Comunicação de resultados ao cliente & 31 & 21 \\
\hline Consulta das pessoas certas para avançar & 29 & 17 \\
\hline "Workshops" com redefinição de conteúdo & 28 & 17 \\
\hline
\end{tabular}




\begin{tabular}{|l|c|c|}
\hline Delegação de objetivos na fase inicial do projeto & 27 & 17 \\
\hline Assistência aberta do cliente & 26 & 14 \\
\hline $\begin{array}{l}\text { Controlo prático da infraestrutura estratégica em reuniões de } \\
\text { processo }\end{array}$ & 26 & 10 \\
\hline Criação de estruturas de trabalho & 25 & 10 \\
\hline Reuniões para mudança de orientação estratégica & 20 & 17 \\
\hline Análise prática, planeamento e mediação em “workshops" & 20 & 14 \\
\hline Acesso direto e aberto a executivos séniores & 20 & 11 \\
\hline Providenciar e definir boa informação para consultores séniores & 19 & 19 \\
\hline $\begin{array}{l}\text { Reunião de término do processo e fórum de apresentação de } \\
\text { resultados }\end{array}$ & 18 & 5 \\
\hline Reunião de direção para tomada de decisão final & 18 & 15 \\
\hline Reuniões preliminares de informação & 18 & 11 \\
\hline Análise de práticas de rumos de ação e controlo do processo & 14 & 8 \\
\hline $\begin{array}{l}\text { Mudança no processo estratégico baseado na contratação de } \\
\text { especialistas }\end{array}$ & 13 & 4 \\
\hline $\begin{array}{l}\text { Desenvolvimento do trabalho com base em pessoal da preferência } \\
\text { do cliente }\end{array}$ & 11 & 2 \\
\hline Análises mediadoras e de reorganização em “workshops” & 11 & 11 \\
\hline Conceber diferentes possibilidades de trabalho & 8 & 6 \\
\hline Realização de trabalho de casa como uma estrutura de trabalho & 7 & 0 \\
\hline Resolução de um problema particular & 6 & 1 \\
\hline Autoinformação dos consultores & 6 & 1 \\
\hline Fórum de controlo em reuniões de processo & 4 & 0 \\
\hline
\end{tabular}

Fonte: Elaboração dos Autores

Analisando estes dados ao nível qualitativo, os resultados das entrevistas apontam para um conjunto de "práticas" e "praxis" idênticas às identificadas na análise quantitativa. Ao nível das "práticas" identificadas pelos clientes como mais importantes para o sucesso de um trabalho de consultoria, destacam-se, as capacidades técnicas, o cumprimento de prazos e a alocação certa de recursos, sendo na vertente dos consultores realçada a capacidade de comunicação. No que diz respeito às "praxis", são enfatizadas pelos consultores como fulcrais para o desenvolvimento do processo estratégico, os "workshops", as reuniões durante projetos, as reuniões de apresentação e o "reporting" permanente de ações, sendo destacadas pelos clientes as reuniões durante projetos.

A análise de conteúdo realizada com dos dados recolhidos nas entrevistas, permitiu verificar a importância atribuída por consultores e clientes (gestores de PME) relativamente (1) à inclusão de especialista de outras áreas de atividade no desenvolvimento de projetos de consultoria de gestão, (2) à identificação das fases de diagnóstico, inferência, tratamento, mas também de acompanhamento/avaliação/reavaliação como a base do conhecimento especializado no setor [( incorporando-se nesta perspetiva ao modelo de Fincham et al. (2008) a fase de acompanhamento/avaliação/reavaliação)] e, (3) a um conjunto de práticas formais e informais que devem ser tidas simultaneamente em consideração no desenvolvimento de um projeto estratégico no setor de consultoria de gestão, devendo tal como mencionado pelos entrevistados, existir sempre uma combinação das duas, isto porque a prática informal pode muitas vezes ajudar a obter resultados que provavelmente a prática formal por si só não permitiria. 


\section{Discussão de resultados}

Jarzabkowski e Spee (2009) relacionando três diferentes tipos de práticos (indivíduos internos, práticos agregados e práticos agregados externos) e os três diferentes níveis de "praxis" desenvolveram a já apresentada tipologia dos nove domínios da pesquisa da "estratégia-comoprática", sendo que cada um dos domínios representa um possível campo de pesquisa nesta área.Tal como pode ser visualizado através da tabela, um facto que se retém é que os estudos sobre os domínios $\mathrm{H}$ e G, têm sofrido de uma clara falta de atenção por parte da investigação académica, não tendo sido de todo uma área muito trabalhada pelos investigadores da estratégia. Esta mesma constatação fica demonstrada ao nível das "practices", sendo importante ilustrar neste contexto um conjunto de práticas cognitivas, comportamentais, processuais e motivacionais das tarefas rotineiras do trabalho de formulação e implementação da estratégia ao nível micro (Jarzabkowski et al.,2007).

Desta forma, com o objetivo de contribuir para a construção da literatura no campo de pesquisa da estratégia-como-prática, foi importante identificar as "práticas" e "praxis" estratégicas mais importantes para o sucesso de um processo de consultoria de gestão na vertente de análise de consultores e clientes, identificar o tipo de práticas a estas subjacentes (formais ${ }^{2}$ e/ou informais ${ }^{3}$ ), avaliar o grau de importância da inclusão de especialistas de outras áreas no desenvolvimento de projetos de consultoria de gestão e, identificar as diferentes fases que devem ser consideradas como a base do conhecimento especializado no setor de consultoria de gestão (Figura 4), tendo a sua finalidade o objetivo de conseguir que os profissionais da área de consultoria de gestão aprendam a adaptar e fazer uso deles nos seus contextos particulares.

O desenvolvimento de um processo de consultoria de gestão deve ser centrado sobre uma estrutura de conhecimentos onde se incluem a fase de diagnóstico, inferência, tratamento e acompanhamento/avaliação/reavaliação. Este ciclo de desenvolvimento num processo de consultoria de gestão é o que se pode designar como os quatro modos de um conhecimento especializado. Neste contexto, a construção do conhecimento deve funcionar como uma rede de trabalho e agir como um canal de informação onde aspetos técnicos e práticos se devem fundir para construir uma fonte inteligente de conhecimentos que deve ser utilizada pelos consultores face às complexas e diferentes contingências existentes na condução de cada projeto.

\footnotetext{
${ }^{2}$ Designando-se como formais todos os processos de exploração planeados com antecedência (Cummings e Wilson, 2003) - reuniões preliminares, reuniões exploratórias, reuniões de "kick off", apresentação de metodologias de trabalho, redefinição de estratégias, reuniões de equipas de trabalho, identificação de equipas de trabalho, apresentação de relatórios e/ou formulários, "workshops", sessões de acompanhamento ou levantamentos estratégicos como a análise SWOT, PEST, ABC ou BCG

${ }^{3}$ Como informais devem ser consideradas todas aquelas que aparecem junto dos procedimentos formais e que ocorrem livremente no tempo e no espaço, sem relações explícitas para o sistema organizacional (Cummings e Wilson, 2003) - jantares e visitas de campo, troca de ideias de forma a gerar focos de discussão, telefonemas, esclarecimentos de dúvidas, conversas informais na tentativa de se perceber o que efetivamente se pretende, despertar de uma oportunidade negocial, "feelings", criação de relações de confiança, apresentação de problemas com carácter informal nos primeiros 15 minutos de reuniões, almoços ou um café que possa indiciar algo que não está na formalidade de um relatório
} 
Figura 4: Identificação das "practices" e "praxis" estratégicas mais importantes para o sucesso de um processo de consultoria de gestão na vertente de análise de consultores e clientes

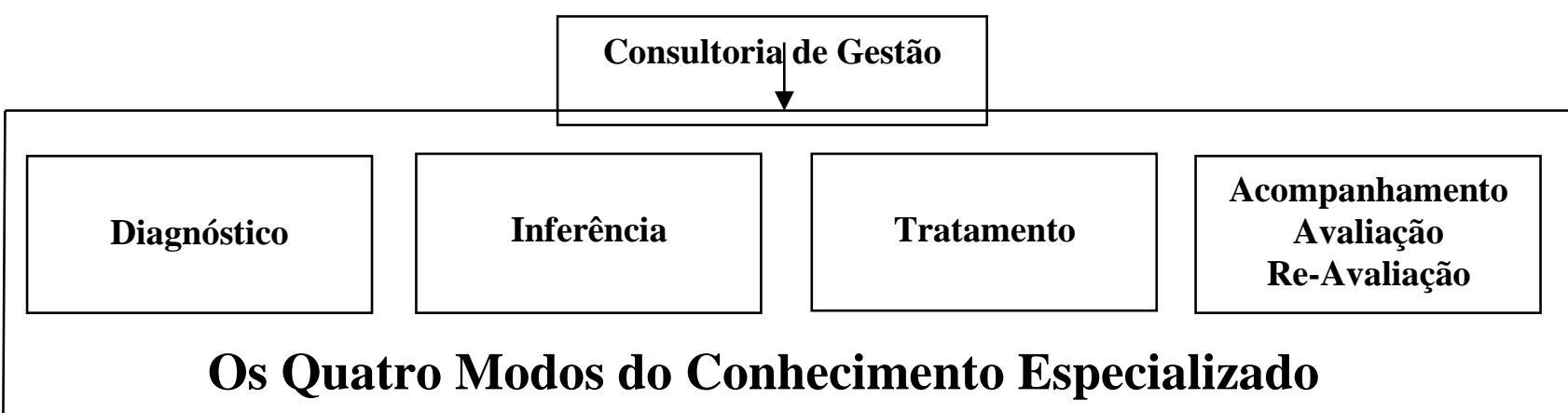

"Zerar" Conhecimentos na Fase de Diagnóstico
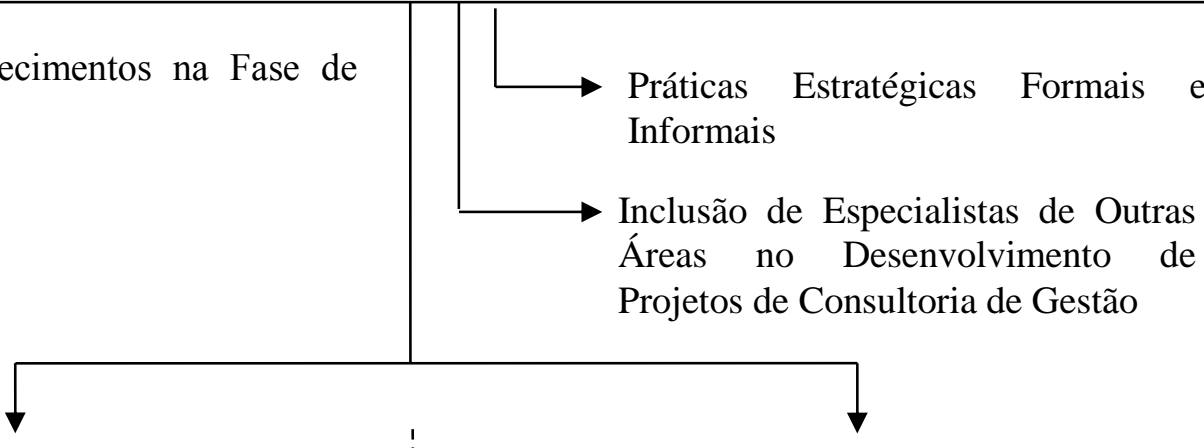

"Practices" Estratégicas a Considerar

\section{Ótica dos Consultores}

- Capacidade de influenciar uma decisão estratégica

- Persuasão, influência e assertividade

- Mente aberta para novas visões ou soluções

- Honestidade de abordagem

- Criação de relações de confiança

- Capacidade de se colocar na pele dos clientes

Flexibilidade adaptabilidade no processo de decisão

- Boa capacidade de interação pessoal

- Capacidade de comunicação

\section{Ótica dos Clientes}

- Capacidade de influenciar uma decisão estratégica

- Persuasão, influência e assertividade

- Mente aberta para novas visões ou soluções

- Honestidade de abordagem

- Criação de relações de confiança

- Capacidade de se colocar na pele dos clientes

- Flexibilidade
adaptabilidade no processo de
decisão
- $\underline{\text { Capacidades técnicas }}$
- $\underline{\text { Cumprimento de prazos }}$
- $\underline{\text { Alocação certa de recursos }}$

"Praxis" Estratégicas a Considerar

\section{Ótica dos Consultores}

- Reunião semanal ao nível da análise, planeamento e implementação

- Reunião de processo para início do estudo

- Conhecer os interesses, valores, atitudes e visões do cliente

- Identificação do cliente e do negócio

- Metodologias de trabalho

- Bom senso para/e com os clientes

- "Workshops"

- Construção heterogénea do grupo de trabalho

- "Reporting" permanente de ações

\section{Ótica dos Clientes}

- Reunião semanal ao nível da análise, planeamento e implementação

- Reunião de processo para início do estudo

- Conhecer os interesses, valores, atitudes e visões do cliente

- Identificação do cliente e do negócio

- Metodologias de trabalho

- Comunicação de resultados ao cliente

- Providenciação e definição de boa informação para consultores séniores

Fonte: Elaboração dos Autores

Se analisarmos o efeito de polinização como resultado da construção de um conhecimento coletivo [(sendo desde logo nesta perspetiva posto em causa o conhecimento especializado de um dado setor de Fincham et al. (2008)] e considerarmos que ao se iniciar uma ação de 
consultoria tudo aquilo que um consultor sabe deve ser esquecido sob pena de ficar viciado no seu próprio raciocínio, o maior trunfo que o consultor pode deter deve ser o de questionar, perceber aquilo que os clientes precisam antes deles próprios e munir-se de meios que permitam apoiar as empresas na implementação das ações que lhes são propostas, incluindo sempre que se mostrar necessário e em qualquer fase do processo, especialistas de outras áreas de atividade que os possam auxiliar no desenvolvimento dos seus trabalhos.

Face às múltiplas especificidades deste setor, a construção de um modelo que permita identificar as "practices" e "praxis" a considerar no desenvolvimento da atividade estratégica deve ainda evoluir considerando sempre um conjunto de práticas formais, mas também de práticas informais, ficando ilustrado nesta investigação que as duas devem aparecer em simultâneo no decorrer de toda a atividade estratégica, sendo ambas importantes no desenvolvimento de cada fase do processo.

Contextualizando a análise em termos das "practices" e "praxis" às quais se deve dar especial atenção em termos da transferência do conhecimento para o sucesso do trabalho estratégico dos consultores, ao nível das "practices" devem destacar-se, (1) a capacidade de influenciar uma decisão estratégica, (2) a persuasão, influência e assertividade, (3) ter mente aberta para novas visões e soluções, (4) a honestidade de abordagem, (5) a criação de relações de confiança, (6) a capacidade que os consultores devem ter de se colocar na pele dos clientes, (7) as suas capacidades técnicas, (8) o cumprimento de prazos, (9) a alocação certa dos recursos humanos e, (10) flexibilidade e adaptabilidade no processo de decisão. Em termos das "praxis" são tidas por gestores (clientes) como fundamentais no processo, (1) a reunião semanal ao nível da análise, planeamento e implementação, (2) a reunião de processo para início do estudo, (3) o conhecer dos interesses, valores, atitudes e visões do cliente, (4) a identificação do cliente e do negócio, (5) a comunicação de resultados, (6) providenciar e definir informação adequada para consultores seniores e, (7) as metodologias de trabalho.

Os resultados aqui apresentados pretendem desta forma ilustrar um conjunto de micro rotinas práticas e de "praxis" que permitam clarificar o caminho de ação e interação dos consultores de gestão no desenvolvimento do seu trabalho estratégico, trazendo simultaneamente para o campo de investigação da estratégia-como-prática um conjunto de ações e orientações estratégicas para os consultores de gestão que os possam conduzir eficientemente na direção de maiores índices de desempenho organizacional (Jarzabkowsk, 2005; Whittington, 2006; Jarzabkowski et al., 2007; Jarzabkowski e Spee, 2009).

\section{Considerações Finais}

É importante no entanto que se tenha em conta que as constatações apresentadas neste estudo, resultam de limitações inerentes a uma investigação reduzida em termos de tamanho da amostra (inquiridos) e do fato de reproduzir resultados de um determinado contexto (PME), num determinado país (Portugal). Neste sentido, em termos de validade externa, ou seja, da possibilidade de generalizar os resultados encontrados a outros contextos ou amostras, embora este estudo tenha vindo reforçar alguma da teoria já existente relativamente ao conceito de consultoria de gestão, este tratou-se apenas de um estudo exploratório que não pode ser generalizado ou representativo. 
Por outro lado, apesar das fontes secundárias terem sido utilizadas e outras análises tenham sido elaboradas para completar os resultados, também este fator não pode justificar que os resultados aqui apresentados possam ser vistos como necessariamente generalizáveis em termos da prática de consultoria. Outra das limitações esteve relacionada com a impossibilidade de observar "in loco" interações cliente-consultor e, portanto, as consequentes particularidades de problemas, ideias e técnicas que poderiam resultar desta mesma interação. Assim, ainda que este artigo vise fundamentalmente combater a falta de estudos sobre o setor de consultoria de gestão, é necessário continuar a penetrar neste mercado e perceber esta atividade, trazendo para o campo académico um volume considerável de informação que traga acima de tudo originalidade e contribua de forma concreta para o desenvolvimento deste campo de pesquisa.

Uma das formas de responder a este "gap", é através da focalização, estabelecimento e institucionalização das práticas e atividades utilizadas no setor de consultoria de gestão e das implicações destas em termos de resultados organizacionais, procurando a obtenção de um conjunto de novos desenvolvimentos na construção da literatura com a introdução de novas perspetivas teóricas e empíricas nesta área. Em suma, é importante estender estes estudos a uma base mais profunda relativamente a todas estas matérias, a fim de que seja explorado o futuro da natureza das práticas, das atividades, das etapas e dos relacionamentos no setor de consultoria de gestão, podendo futuras pesquisas incluir neste sentido a construção de um modelo que permita relacionar todas estas variáveis, a fim de que se consiga identificar quais delas é mais determinante para o sucesso de um projeto de consultoria de gestão. A tarefa contínua do estudo do setor não deve esquecer também o impacto que tudo isto pode ter a jusante da cadeia, nomeadamente ao nível grau de satisfação das empresas cliente face aos trabalhos desenvolvidos pelos consultores e de quais as sugestões por si aconselhadas para a melhoria dos desempenhos que se podem vir a obter. 


\section{REFERÊNCIAS}

Balogun, J. e G. Johnson (2005), Intended strategies to unintended outcomes: the impact of change recipient sense making, OrganizationStudies26, 1573-1601.

Carmo, H. e Ferreira, M. (1998), Metodologia da Investigação: Guia para Auto-aprendizagem, Universidade Aberta, Lisboa.

Fincham, R., T. Clark., K. Handley e A. Sturdy (2008), Configuring expert knowledge: the consultant as sector specialist, Journal of Organizational Behaviour 29 (8), 1145-1160.

Jarzabkowski, P. (2004), Strategy-as-practice: Recursiveness, adaptation, and practices-inuse, Organization Studies 25 (4), 529-560.

Jarzabkowski, P. (2005), Strategy-as-Practice: An Activity Based Approach, Sage: London.

Jarzabkowski, P. e P. Spee (2009), Strategy-as-practice: A review and future directions for the field, International Journal of Management Reviews 11 (1), 69-95.

Jarzabkowski, P., J. Balogun e D. Seidl (2007), Strategizing: The challenges of a practice perspective, Human Relations 60 (1), 5-27.

Jarzabkowski, P. e R. Whittington (2008), Hard to disagree, mostly, Strategic Organization6, 101-106.

Johnson, G., L. Melin, e R. Whittington (2003), Micro strategy and strategizing: Towards an activity-based view, Journal of Management Studies 40 (1), 3-22.

Johnson, G., A. Langley, L. Meline R. Whittington (2007), Strategy as Practice: Research Directionsand Resources, Cambridge University Press: Cambridge.

Laine, P. e E. Vaara (2007), Struggling over subjectivity: a discursive analysis of strategic development in an engineering group, Human Relations 60, 29-58

Lounsbury, M. e E. Crumley (2007), New practice creation: An institutional perspective on innovation,Organization Studies28, 993-1012.

Lundgren, M. e M. Blom (2009), The Practice of Strategy Consultants, 25 th Egos Colloquium, Barcelona (Julho 2-4).

Mazza, C. e J. Alvarez (2000), Haute couture and prêt-à-porter: The popular press and the diffusion of management practices, Organization Studies21, 567-588.

Melin, L. e M. Nordqvist (2007), The reflexive dynamics of institutionalization: The case of the family business,Strategic Organization5, 321-333.

Seidl, D. (2007), General strategy concepts and the ecology of strategy discourses: A systematic-discursive perspective, Organization Studies 28, 197-218. 
Sillince, J. e F. Mueller (2007), Switching strategic perspective: The reframing of accounts of responsibility, Organization Studies28, 155-176.

Sminia, H. (2005), Strategy formation as layered discussion, Scandinavian Journal of Management 21, 267-291.

Sztompka, P. (1991), Society in Action: the Theory of Social Becoming, Polity Press: Cambridge:

Reckwitz, A. (2002), Towards a theory of social practice: A development in cultural theorizing, European Journal of Social Theory 5, 243-262.

Sturdy, A., M. Schwarz e A. Spicer (2006), Guess who is coming for dinner? Structures and uses of liminality in strategic management consultancy, Human Relations 59, 929-960.

Werr, A. e A. Styhre (2002), Management consultants friend or foe?, International Studies of Management \& Organization 32 (4), 43-66.

Whittington, R. (1996), Strategy-as-practice, Long Range Planning 29 (5), 731-735.

Whittington, R (2002), Practice perspectives on strategy: Unifying and developing a field,Academy of Management Conference: Denver.

Whittington, R. (2006), Completing the practice turn in strategy research, Organization Studies 27 (5), 613-634. 